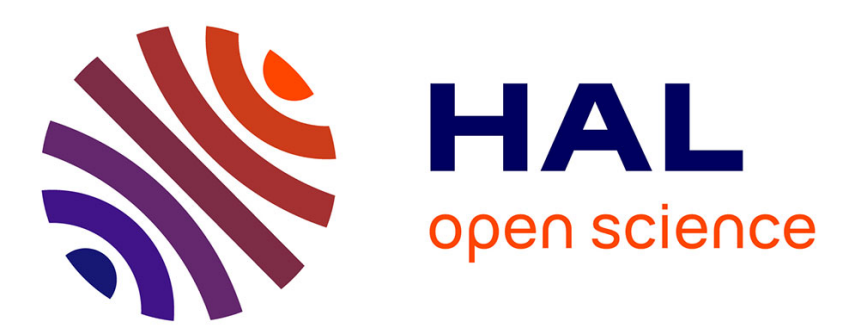

\title{
Correctness Analysis of MPI-3 Non-Blocking Communications in PARCOACH
}

Julien Jaeger, Emmanuelle Saillard, Patrick Carribault, Denis Barthou

\section{To cite this version:}

Julien Jaeger, Emmanuelle Saillard, Patrick Carribault, Denis Barthou. Correctness Analysis of MPI3 Non-Blocking Communications in PARCOACH. European MPI Users' Group Meeting, Sep 2015, Bordeaux, France. 10.1145/1235 . hal-01252321

\section{HAL Id: hal-01252321 \\ https://hal.inria.fr/hal-01252321}

Submitted on 7 Jan 2016

HAL is a multi-disciplinary open access archive for the deposit and dissemination of scientific research documents, whether they are published or not. The documents may come from teaching and research institutions in France or abroad, or from public or private research centers.
L'archive ouverte pluridisciplinaire HAL, est destinée au dépôt et à la diffusion de documents scientifiques de niveau recherche, publiés ou non, émanant des établissements d'enseignement et de recherche français ou étrangers, des laboratoires publics ou privés. 


\section{Correctness Analysis of MPI-3 Non-Blocking Communications in PARCOACH}

\author{
Julien Jaeger \\ CEA, DAM, DIF \\ F-91297 Arpajon, FRANCE \\ julien.jaeger@cea.fr \\ Patrick Carribault \\ CEA, DAM, DIF \\ F-91297 Arpajon, FRANCE \\ patrick.carribault@cea.fr
}

\author{
Emmanuelle Saillard \\ CEA, DAM, DIF \\ F-91297 Arpajon, FRANCE \\ emmanuelle.saillard.ocre@cea.fr \\ Denis Barthou \\ Bordeaux Institute of \\ Technology \\ LaBRI/INRIA \\ Bordeaux, FRANCE \\ denis.barthou@inria.fr
}

\begin{abstract}
MPI-3 provide functions for non-blocking collectives. To help programmers introduce non-blocking collectives to existing MPI programs, we improve the PARCOACH tool for checking correctness of MPI call sequences. These enhancements focus on correct call sequences of all flavor of collective calls, and on the presence of completion calls for all nonblocking communications. The evaluation shows an overhead under $10 \%$ of original compilation time.
\end{abstract}

\section{Keywords}

MPI, Non-blocking collectives, checker, static analysis

\section{INTRODUCTION}

Most parallel scientific applications rely on the distributedmemory specification called MPI (Message Passing Interface) to efficiently exploit a supercomputer and reach high parallel performance. This programming model proposes point-to-point and collective communications to transfer messages between the different processes. Furthermore, the first specification provides the notion of non-blocking point-topoint communications.

Non-Blocking Point-To-Point Communications and Completion Calls: Non-blocking point-to-point communication allows to overlap communication and computation and thus to leverage hardware parallelism. Several studies [2] have shown that the performance of parallel applications can be significantly enhanced with overlapping techniques. A call to a non-blocking point-to-point communication initiates an action without completing it. Another function, called completion call is necessary to finish the action. At the end of this completion call, the runtime ensures

ACM ISBN 978-1-4503-2138-9.

DOI: $10.1145 / 1235$ that it is safe for the application to reuse the communication buffers. One can distinguish two types of completion calls: completion calls that complete only one non-blocking operation ( functions MPI_\{Wait, Waitany, Test, Testany\}, referred to as unitary completion call or ucc) and completion calls checking in a list of operations all the ones that are done (functions MPI_\{Waitall, Waitsome, Testall, Testsome\}, referred to as multi completion call or mcc).

Collective Communications: Collective communications are an important part of parallel scientific computing [1]. The non-blocking collectives provided in MPI-3 API combine the efficient algorithms of collectives operations with the overlapping benefits of non-blocking communications. The non-blocking collective model is similar to the one proposed by non-blocking point-to-point communications. The main difference with point-to-point communications is that blocking collectives can not be matched to their non-blocking counterparts. The ordering of collectives between blocking and non-blocking collectives, and between non-blocking collectives, is very important. If one process calls a non-blocking collective, the remaining processes should also call the same function before initiating any other collective.

In this paper, we introduce an evolution of the PAR$\mathrm{COACH}$ [3] tool with a new static analysis allowing to debug MPI non-blocking call sequences.

\section{ANALYSIS OF NON-BLOCKING COM- MUNICATIONS}

To tackle the issues pointed out in Section 1, we propose two new analyses: (i) a new static pass to check if the number of completion calls may match non-blocking call (either collective or point-to-point) and (ii) a static analysis to check that the sequence of all flavor of collective is the same along all possible execution paths. These analyses have been implemented inside PARCOACH [3], a tool proposing a twophase analysis to detect incorrect collective patterns in MPI programs. It combines a static analysis identifying the reduced set of problematic collectives with a selective instrumentation on the corresponding nodes in the Control Flow Graph (CFG) of the function to handle the deadlocks. 
Matching Non-blocking Communication with Completion Calls: With this new pass, we determine if each non-blocking operation, either point-to-point or collective, can be matched to a completion call. For a path to be valid, each non-blocking operation should correspond to a unitary completion call or to a multi completion call. The exact matching would require to execute the code, checking all arguments passing and memory movements. Statically, we make the following assumption: a completion call always matches a previous pending non-blocking operation, if any. Within this assumption and for each basic block of the analyzed function, the number of pending non-blocking communications (called $p n b$ ) is computed, using an interval notation, following Algorithm 1 . The lower bound $(l b)$ corresponds to the number of pending communications when $m c c$ statements complete all the previous non-blocking communications. The upper bound $(u b)$ corresponds to the number of pending communications case when mcc statements only complete one of the previous non-blocking communication. Given this approach, a function is incorrect (completion calls missing) if the lower bound of the pnb for the function exit is greater than 0 . The function may be incorrect if the upper bound of this $p n b$ is greater than 0 .

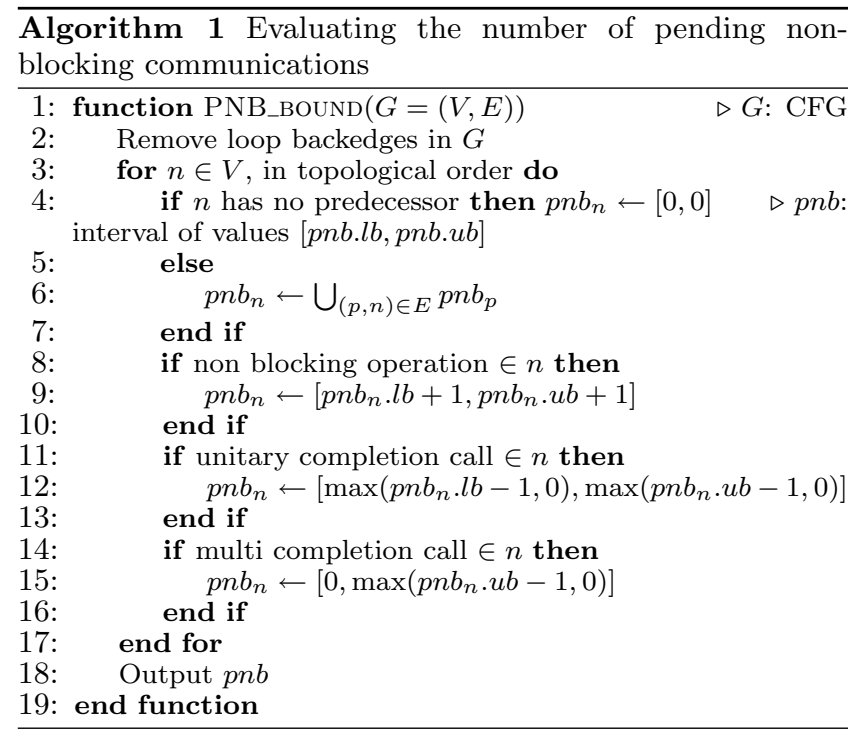

\section{Extending PARCOACH with Non-Blocking Seman-} tics:

In Section 1, non-blocking collectives have been shown to share the same constraint as blocking collectives when used together. Once this behavior is identified, supporting nonblocking collectives in the original analysis of PARCOACH is straightforward: the syntactic parser of collective names has to be enhanced with non-blocking ones. Since PARCOACH uses the output list of this step to instrument the problematic nodes, our enhancement will directly benefit from the instrumentation. PARCOACH will then be able to insert functions for runtime checking before all flavor of collectives, including non-blocking ones.

\section{RESULTS}

We tested our two static analyses on the Intel MPI Benchmarks version 4.0 (IMB) and a microbenchmarks suite of unitary tests we created (NBC-bench). These results were

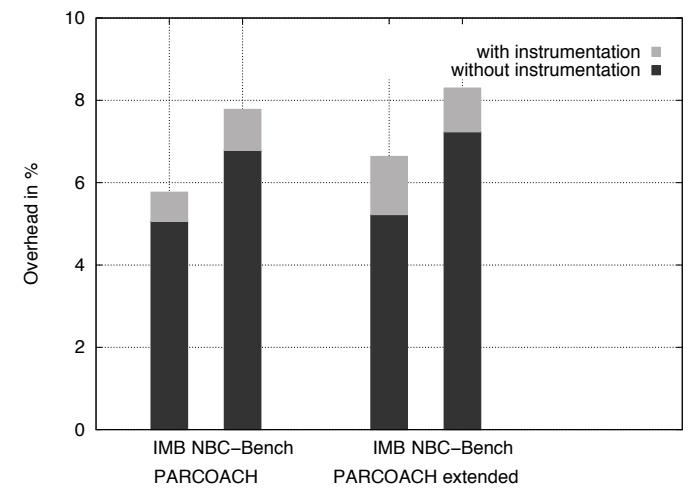

Figure 1: Overhead of average compilation time with and without verification code generation

computed and averaged with MPICH 3.1.4.

Figure 1 highlights the compilation-time overhead obtained with and without static instrumentation (validation functions insertions) on the two sets of benchmarks. For each test, the overhead of the original PARCOACH analysis, for blocking MPI collective calls, and our improved analysis, checking both blocking and non-blocking MPI collective calls, are provided. The black bars display the original overheads, and the grey bars show the additional overhead created by the addition of non-blocking collectives in the static analysis. Our addition to the checking of collective only bring an extra $1 \%$ to the overhead, which remains under $9 \%$ of the total compilation time.

\section{CONCLUSION}

In this paper, we extended the static analysis of PAR$\mathrm{COACH}$, originally designed for checking blocking collective calls, to include non-blocking collectives. A second static analysis was included to also check the completion of all non-blocking communications issued in an MPI program. The designed algorithms allow to find if non-blocking calls are not completed in a function, and return the list of problematic nodes for issuing warnings to the developer. This addition brought a small extra overhead compared to the original analysis, and the complete compilation time with the new analyses being less than $10 \%$ higher than initial compilation time.

\section{REFERENCES}

[1] D. Dureau and G. Poëtte. Hybrid parallel programming models for amr neutron monte-carlo transport. In SNA + MC 2013-Joint International Conference on Supercomputing in Nuclear Applications + Monte Carlo, page 04202. EDP Sciences, 2014.

[2] T. Hoefler, P. Gottschling, A. Lumsdaine, and W. Rehm. Optimizing a conjugate gradient solver with non-blocking collective operations. Elsevier Journal of Parallel Computing (PARCO), 33(9):624 - 633, 2007. Selected Papers from EuroPVM/MPI 2006.

[3] E. Saillard, P. Carribault, and D. Barthou. Parcoach: Combining static and dynamic validation of MPI collective communications. Intl. Journal on High Performance Computing Applications (IJHPCA), 28(4):425-434, 2014. 